\title{
Study of the Underlying Event with the CMS detector at the LHC
}

\author{
Andrea Lucaroni \\ on behalf of the CMS collaboration \\ Universitá degli Studi di Perugia \& INFN Perugia \\ E-mail: andrea.lucaroni@cern.ch
}

\begin{abstract}
The underlying event in pp interactions at both $900 \mathrm{GeV}$ and $7 \mathrm{TeV}$ is studied exploiting the performances of the powerful CMS tracking system. Charged multiplicity and energy densities are measured in charged jet events concentrating in the regions perpendicular to the plane of the hard 2-to-2 scattering which includes the beam and the jet directions. A significant underlying event activity increase with the jet transverse momentum is reported, confirming the so called "Pedestal Effect". A factor two increase of the Underlying activity is observed at $7 \mathrm{TeV}$ with respect to 900 $\mathrm{GeV}$. These studies allow to discriminate between various QCD Monte Carlo models which correctly reproduce the Tevatron Underlying Event phenomenology but achieve different predictions at different energies. The knowledge of some key aspects of the models is improved, in particular for what concerns the energy dependency of the phenomenological parameters regulating the minimal scale of the Multiple Parton Interactions.
\end{abstract}

35th International Conference of High Energy Physics - ICHEP2010,

July 22-28, 2010

Paris France 


\section{Introduction}

The Underlying Event (UE) in a hard scattering process is everything accompanying an event but the hard scattering component of the collision. In this study we have measured the UE activity using two samples collected by the CMS detector [1]. The first one is referred to the 2009 runs at $900 \mathrm{GeV}$ related to an integrated luminosity of $1 \mu \mathrm{b}^{-1}$ [2]. The second one was collected at $7 \mathrm{TeV}$ with the first LHC runs of the 2010 and is corresponding to an integrated luminosity of $1 \mathrm{nb}^{-1}$ [3].

The analysis uses the "jet" structure of the hard proton-proton collision to experimentally study the UE activity. Track-jets are reconstructed from tracks using a cone algorithm. The direction of the hard scattering is assumed correlated with the leading track-jet (ltj) direction and is used to isolate regions of $\eta-\phi$ space that are maximally sensitive to the UE. Tracks are used to account the activity in the transverse region to the track-jet direction, defined by $\pi / 3<\left|\phi_{t r a c k}-\phi_{l t j}\right|<2 \pi / 3$. The $p_{T}$ of the leading track-jet defines the energy scale of the event.

The UE activity is studied looking at the charged track multiplicity and scalar sum of the $p_{T}$ as a function of leading track-jet in the transverse region. The data are compared with various tunes of PYTHIA [4]. Different models make use of different values of the parameters in the implementation of the regularization of the formal $1 / p_{T}$ divergence of the leading order parton scattering amplitude, as the final state parton transverse momentum $p_{T}$ in the limit to zero. In PYTHIA this divergence is regularized through the replacement $1 / p_{T}^{4} \rightarrow 1 /\left(p_{T 0}^{2}+p_{T}^{2}\right)^{2}$. In which the transverse momentum $p_{T 0}$ is the cross-section cut-off and it is parameterized as $p_{T 0}(\sqrt{s})=$ $p_{T 0}\left(\sqrt{s_{0}}\right)\left(\sqrt{s} / \sqrt{s_{0}}\right)^{\varepsilon}$, where $\sqrt{s_{0}}$ is the reference energy at which $p_{T 0}$ is determined and $\varepsilon$ is a parameter describing the energy dependence. $p_{T 0}$ governs the amount of the multiple parton interactions (MPI) in the event, the main component of the UE activity.

\section{Event and track selection}

The adopted trigger is defined as the coincidence of both Beam Pick-up Timing for eXperiments [5] with a hit in the Beam Scintillator Counters and requiring a primary vertex. Event and track selection strategies are similar at the considered center of mass energies. A track is selected for the UE analysis if it originates from the primary vertex and is reconstructed with transverse momentum $p_{T}>0.5 \mathrm{GeV} / c$ and pseudorapidity $|\eta|<2$. To decrease contamination by secondary tracks from decays of long-lived particles and photon conversion, the distance of closest approach between track and primary vertex is required. The leading track-jet is reconstructed with the tracks selected using the SISCone algorithm [6]. Possible sources of systematic uncertainties are considered, such as tracks selection criteria, tracker alignment, tracker material content, back ground contamination, trigger condition and variation of the beam spot condition. An average value similar for the two analysis of $2 \%$ relative systematic error has been computed.

\section{Results}

The charged multiplicity in Figure 1, and the $p_{T}$ scalar sum (here not shown) as a function of track-jet $p_{T}$ (the interaction scale) shows the same features in data and MC: a fast rise due to MPI at low $p_{T}$ followed by a slower increase due to radiation above $5 \mathrm{GeV} / c$ and $8 \mathrm{GeV} / c$ for 0.9 and 
$7 \mathrm{TeV}$ respectively. Figure 1 (right) shows CMS data on the ratio between $7 \mathrm{TeV}$ and $900 \mathrm{GeV}$ for the transverse charged particle density. DW tune provides the best description for both centre of mass energies.
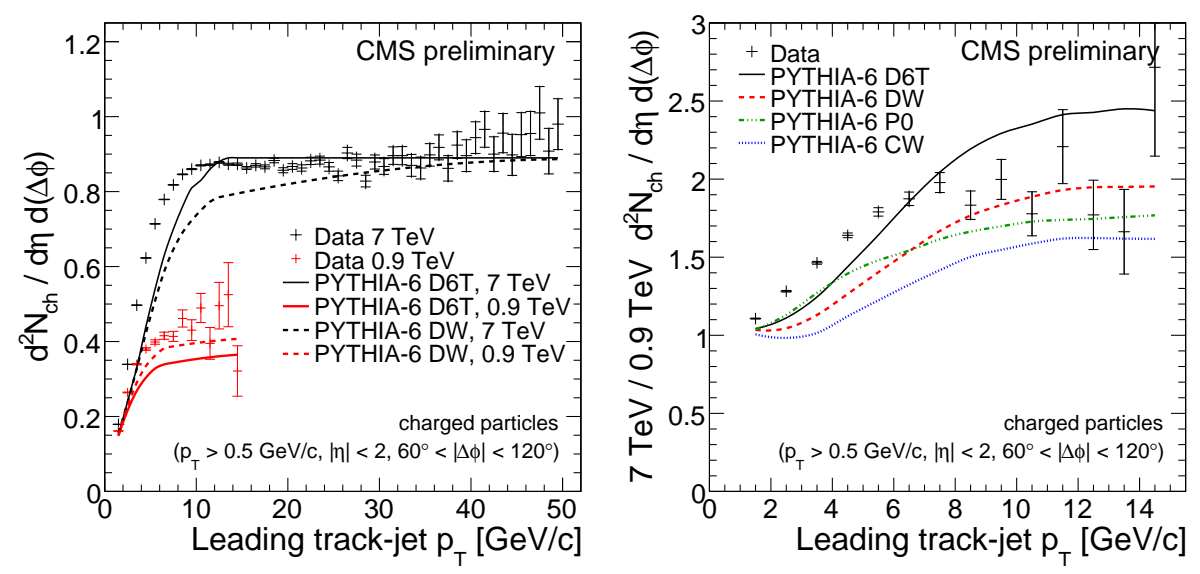

Figure 1: Left plot shows the average multiplicity as a function of the leading track jet $p_{T}$, for data at $\sqrt{s}=0.9 \mathrm{TeV}$ and $\sqrt{s}=7 \mathrm{TeV}$, right plot the ratio of the average values at 7 and $0.9 \mathrm{TeV}$.

\section{Conclusions}

A study has been presented on the Underlying Event activity in proton-proton interactions at both $900 \mathrm{GeV}$ and $7 \mathrm{TeV}$. A significant activity increase with the jet transverse momentum is reported, confirming the so called "Pedestal Effect". A factor two increase of the Underlying Event activity is observed at $7 \mathrm{TeV}$ with respect to $900 \mathrm{GeV}$. The comparison between data collected and the MC predictions shows a strong $\sqrt{s}$ dependence of $p_{T 0}$, as in tune DW $(\varepsilon=0.25)$, compared to tune D6T $(\varepsilon=0.16)$.

\section{References}

[1] CMS Collaboration, "The CMS experiment at the CERN LHC" JINST 3:S08004 (2008).

[2] CMS Collaboration, "Measurement of the Underlying Event Activity in Proton-Proton Collisions at 0.9 TeV." arXiv:1006.2083 [hep-ex].

[3] CMS Collaboration, "Measurement of the Underlying Event Activity at the LHC with $\sqrt{s}=7 \mathrm{TeV}$ and Comparison with $\sqrt{s}=0.9$ TeV." CMS-QCD-10-010 (2010).

[4] T. Sjostrand, S. Mrenna and P. Z. Skands, "PYTHIA 6.4 Physics and Manual" JHEP 0605 (2006) 026 [arXiv:hep-ph/0603175].

[5] Thomas Aumeyr, "Evaluation of Oscilloscopes for the BPTX Read-Out System - Tests in the ATLAS Trigger Electronics Lab." CMS-IN-2007/049.

[6] G. P. Salam and G. Soyez, "A practical Seedless Infrared-Safe Cone jet algorithm.” JHEP 0705 (2007) 086 [arXiv:0704.0292 [hep-ph]]. 